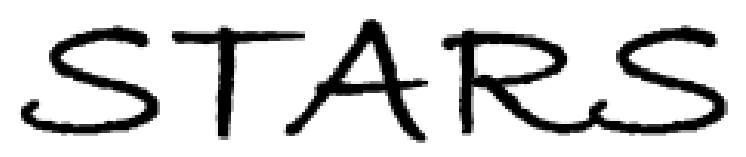

University of Central Florida

STARS

$1-1-2002$

\title{
Spatially resolved microrheology using localized coherence volumes
}

G. Popescu

University of Central Florida
A. Dogariu
University of Central Florida
R. Rajagopalan

Find similar works at: https://stars.library.ucf.edu/facultybib2000

University of Central Florida Libraries http://library.ucf.edu

This Article is brought to you for free and open access by the Faculty Bibliography at STARS. It has been accepted for inclusion in Faculty Bibliography 2000s by an authorized administrator of STARS. For more information, please contactSTARS@ucf.edu.

\section{Recommended Citation}

Popescu, G.; Dogariu, A.; and Rajagopalan, R., "Spatially resolved microrheology using localized coherence volumes" (2002). Faculty Bibliography 2000s. 3407.

https://stars.library.ucf.edu/facultybib2000/3407

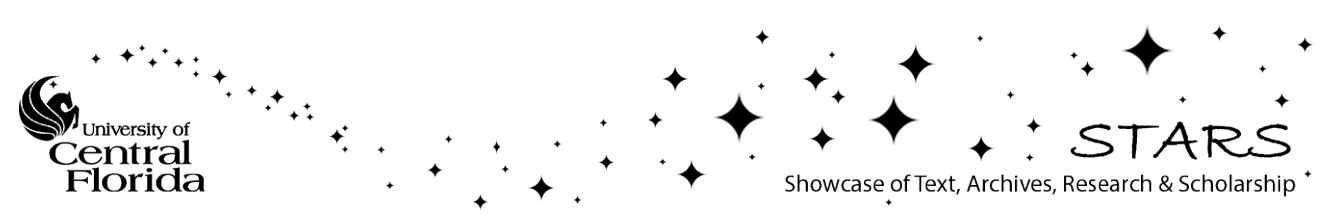




\title{
Spatially resolved microrheology using localized coherence volumes
}

\author{
G. Popescu and A. Dogariu \\ School of Optics, University of Central Florida, Orlando, Florida 32816-2700 \\ R. Rajagopalan \\ Department of Chemical Engineering, University of Florida, Gainesville, Florida 32611-6005 \\ (Received 1 August 2001; revised manuscript received 29 October 2001; published 3 April 2002)
}

\begin{abstract}
We propose an optical technique, based on dynamic light scattering, for investigating the local rheological response of a complex fluid over a frequency range larger than that provided by standard mechanical instrumentation. The low-coherence radiation used in a fiber optics configuration allows the measurements to be confined to a small volume around a tenth of a picoliter. The ability of the method to accurately measure both loss and storage moduli has been tested using both simple Newtonian liquids and viscoelastic, complex fluids. The possibility to monitor liquid-gel transitions in polymer solutions has also been demonstrated. The unique capability of the technique to localize the measurement volume suggests that this novel approach can be used for three-dimensional mapping of rheological properties in heterogeneous systems.
\end{abstract}

DOI: 10.1103/PhysRevE.65.041504

PACS number(s): 83.85.Ei, 82.70.-y, 42.25.Kb

\section{INTRODUCTION}

Soft materials, which include polymer solutions, surfactant solutions, and biological materials, among others, are characterized by complex structures with multiple characteristic time and length scales; thus their response to external strains has a nontrivial time dependence. One of the most important descriptors of these properties is the complex shear modulus $G(t)$, which is typically measured in the frequency domain $\omega$. The real part $G^{\prime}(\omega)$ in the frequency domain describes the elastic (storage) property of the system, while the imaginary part $G^{\prime \prime}(\omega)$ is a measure of the viscous (loss) behavior. The ability to measure locally the mechanical response of a material to an applied shear strain has a variety of potential applications, especially in biology, where the mechanical properties of the cells and intracellular matter are of utmost importance.

Recently a number of techniques have been developed or suggested for probing the rheological properties of complex materials at a microscopic scale [1] —an area that has come to be known as microrheology. Most of the microrheological techniques rely on applying a strain to the fluid through embedded "probe" particles, and the strains result either from the (Brownian) thermal noise imparted through the probe or from a suitable, externally imposed force on the probe. Application of an external force, such as a magnetic field, to the particles may produce a nonlinear response of the fluid since the resulting strain could be substantial, whereas strains resulting from thermally excited probes are quite low and ensure linearity of response in most of the cases. The probe motion is quantified by its mean-squared displacement, which can be obtained by particle tracking [2,3], singleparticle microscopy [4], or dynamic light scattering [5] (e.g., diffusing wave spectroscopy, when multiple scattering is dominant). Note that recording individual particle trajectories typically requires a smaller sample volume than in the case of diffusing wave spectroscopy (DWS), in which the measurements are performed over an ensemble of particles. On the other hand, dynamic light scattering (DLS) tech- niques have the advantage of providing an inherent average over the particle ensemble, while single-particle techniques require successive measurements in order to obtain a reliable average. It is worth noting that atomic force microscopy (AFM) can also be used in this context [6]. Here one monitors the thermal fluctuations of the tip of the AFM cantilever instead of following a probe particle. While an AFM allows one to examine microrheology at interfaces (as do particletracking techniques or the dynamic light scattering technique we propose in this paper), the analysis of the AFM data is not straightforward for a number of reasons. Most notable among these are the complicated geometry of the probe (i.e., the AFM tip) and the influence of the intrusive, mechanical behavior of the AFM cantilever itself (and the resulting, often ill-characterized, fluid response) [6].

Microrheological measurements have been reported in the last few years for a number of materials, e.g., colloidal dispersions, polymer solutions, and biological cells and materials. For example, numerous experiments on actin networks have shown nontrivial high-frequency dependence of the shear modulus and models have been proposed to explain such behavior (see, for example, [7-9]). It is commonly agreed that most of the viscoelastic fluids have shear moduli that follow a power-law dependence in the high-frequency region, but the actual value of the exponent appears to vary depending on the materials and, sometimes, the experimental technique used. More recently, it has been shown that cross correlating the thermal motion of pairs of embedded particles offers a more precise and a different way of examining the microrheology of soft condensed matter, suggesting that measurements using previous microrheological techniques may need to be revised or reexamined [10-12]. Therefore, it is of great interest to find complementary investigation methods for high-frequency viscoelastic behavior of complex fluids at microscopic scales.

The paper is organized as follows. We begin with a brief introduction to microrheological measurements and a description of the localized-coherence-volume scattering technique proposed in this paper. Following these, a series of 
experiments and the analyses of the results are described and discussed. These discussions start with a set of experiments on purely viscous fluids in order to show that the experiments lead to the expected behavior for viscous materials [i.e., $G^{\prime \prime}(\omega) \sim \omega$ and $G^{\prime}(\omega) \ll G^{\prime \prime}(\omega)$ ]. Then we discuss the results obtained for a model polymer solution (polyethylene oxide in water), which is known to become sharply viscoelastic as the polymer concentration is increased and passes over to an elastic "gel" at higher polymer concentrations. We then comment on the liquid-to-gel transition as detected by the proposed technique. An important advantage of the proposed localized-coherence-volume technique is its ability to provide spatial maps of variation in viscoelastic properties. We demonstrate this in the final part of the discussions by considering a one-dimensional interface between a viscous fluid and a viscoelastic polymer solution.

\section{MICRORHEOLOGY IN LOCALIZED COHERENCE VOLUMES}

Here, we propose a technique that relies on quantifying the motion of thermal particles embedded in the fluid under investigation. We show that, by combining the properties of partially coherent light that is guided through a single-mode optical fiber, one can extract, from the intensity of the scattered light, the high-frequency limit of the power spectral density of the mean-squared displacements $\left\langle\Delta r^{2}(\omega)\right\rangle$ of the embedded, probe particles. The measurement volume we use is of the order of a tenth of a picoliter, which is about five to six orders of magnitude smaller than the one needed, for instance, in DWS-based techniques. The microrheological information is then obtained using the fluctuation-dissipation theorem that relates $\left\langle\Delta r^{2}(\omega)\right\rangle$ to the loss response $\chi^{\prime \prime}(\omega)$ that characterizes the fluid through

$$
\chi^{\prime \prime}(\omega)=\frac{\omega}{2 k_{B} T}\left\langle\Delta r^{2}(\omega)\right\rangle,
$$

where $k_{B}$ is Boltzmann's constant, and $T$ is the absolute tempeture of the material. Due to causality, the storage (memory) component $\chi^{\prime}(\omega)$ and loss (dissipative) component $\chi^{\prime \prime}(\omega)$ of the response function $\chi(\omega)$ are related through the Kramers-Kronig equation

$$
\chi^{\prime}(\omega)=\frac{2}{\pi} \mathcal{P} \int_{0}^{\infty} \chi^{\prime \prime}(\xi) \frac{\xi}{\xi^{2}-\omega^{2}} d \xi
$$

where $\mathcal{P}$ indicates the principal value integral.

As noted in the introductory section of this paper, we have tested this new technique on a variety of systems with rheological properties ranging from purely viscous behavior to materials with high viscoelasticity. The experimental geometry and arrangement we use here has been successfully applied in the context of standard dynamic light scattering in localized coherence volumes recently [13] to provide a new optical method for investigating the dynamics of random media over a broad range of optical densities. In our technique, by taking advantage of the spatial coherence properties of a single-mode fiber and the limited temporal coherence of a broadband source, we restrict the measurement volume to the end of the optical fiber. The reduced dimensions of the measurement volume allows the technique to provide accurate results for the particle diffusion coefficient and concentration and to circumvent multiple scattering even for optically dense suspensions. The main characteristic of this dynamic light scattering scheme is that the properties of the system under investigation are probed locally, which can reveal detailed information about its morphology. In addition, the use of an optical fiber allows a high degree of experimental flexibility, thereby enhancing the range of applicability of the technique. It is also important to emphasize another significant advantage offered by the elimination of multiple scattering effects in the proposed technique. The analysis of multiple scattering in DWS-based microrheological studies is based on the assumption that the photon path length is Gaussian. In systems in which the scatterers (either naturally present scatterers or embedded probe particles) are not in large enough concentration to justify the Gaussian assumption, DWS-based analyses of the data introduce numerical artifacts in the extracted complex moduli. Such artifacts, when undetected or not accounted for in the analysis of the data, are attributed to the frequency dependence of the material properties of the fluid. In contrast, in the proposed technique, multiple scattering is efficiently eliminated so that the extracted modulus reflects the true property of the material.

In the following we will show how the local loss and elastic response of a complex fluid can be extracted from the response of the fluid to the thermal excitation communicated by the probe particles. The experimental setup is described in more detail elsewhere [13]. Here we provide a brief description for convenience. In the experimental arrangement, lowcoherence radiation emitted by a superluminescent diode is coupled to a single-mode optical fiber, which represents one arm of a $1 \times 2$ fiber coupler. The light output from the coupler illuminates the fluid under investigation. The light backscattered by the probe particles is collected through the same fiber and is fed to a detector, and the signal is then analyzed in the frequency domain using a spectrum analyzer. The signal detected is an interference of two electromagnetic fields: the fluctuating field backscattered by the particles undergoing thermal motion and the static field due to Fresnel reflection at the fiber-fluid interface. Thus the specular reflection amplifies the backscattering signal, which significantly increases the sensitivity of the technique. The intensity fluctuations are detected only from the coherence volume, defined by the coherence length and the transversal dimension of the fiber core, in which the optical fields preserve relative phase correlations [14].

In the experiments reported here, the central wavelength $\lambda$ of the broadband light was $824 \mathrm{~nm}$, while the coherence length $l_{c}$ had a value of $30 \mu \mathrm{m}$. For this geometry, the relationship between the normalized intensity and amplitude autocorrelation functions has the form [13]

$$
g^{(2)}(\tau)=1+\gamma g^{(1)}(\tau)
$$

where $\gamma=2 I_{0} I_{s}^{\mathrm{CV}}\left(I_{0}+I_{s}^{\mathrm{CV}}\right)^{2}$, with $I_{0}$ and $I_{s}^{\mathrm{CV}}$ the total aver- 
age intensities associated with the Fresnel component and the scattered light from the coherence volume (superscript "CV"), respectively. In all the experiments, the photon mean free path in the medium was always much longer than the coherence length of the light used. Thus, within the coherence volume, the single scattering regime applies and the first-order autocorrelation function $g^{(1)}(\tau)$ can be written as

$$
g^{(1)}(\tau)=\exp \left[-\frac{1}{6} q^{2}\left\langle\Delta r^{2}(\tau)\right\rangle\right]
$$

where $q=4 \pi / \lambda$ is the magnitude of the scattering vector associated with our backscattering geometry, and $\left\langle\Delta r^{2}(\tau)\right\rangle$ is the mean-squared displacement of the particles under thermal motion. The quantity measured in the experiment is the power spectrum of the scattered light fluctuations $P(\omega)$, which is the Fourier counterpart of $g^{(2)}(\tau)$. At this point, we make the observation that, in the high-frequency region, $P(\omega)$ is directly related to the power spectral density of the particle displacements $\left\langle\Delta r^{2}(\omega)\right\rangle$ through

$$
P(\omega)=\frac{\gamma q^{2}}{6}\left\langle\Delta r^{2}(\omega)\right\rangle
$$

Equation (5) holds for times of evolution much shorter than the characteristic decaying time of the autocorrelation function, when Eq. (4) can be linearized as $g^{(1)}(\tau)=1$ $-q^{2}\left\langle\Delta r^{2}(\tau)\right\rangle / 6$. This approximation is similar to the first cumulant expansion used in deriving the Laplace relationship between the autocorrelation function and the path length probability distribution in the context of DWS technique [15]. Therefore, it is rather surprising that the microrheological experiments based on the DWS technique provided accurate results even for long times of evolution [5]. Equation (5) is valid in the high-frequency domain, beyond the characteristic width of the power spectrum $P(\omega)$. Under what conditions this approximation holds is illustrated in Sec. III B. With this approximation, it becomes apparent that the highfrequency dependence of the shear modulus $G(\omega)$ can be obtained by combining Eqs. (1), (2), and (5) using the relationship between the response function $\chi(\omega)$ and $G(\omega)$,

$$
G(\omega)=\frac{1}{6 \pi a} \frac{1}{\chi(\omega)}
$$

where $a$ is the radius of the probe particles [16]. Thus, the measurement of the response function $\chi(\omega)$ in the frequency domain allows one to obtain the rheological information directly in the frequency domain (instead of having to resort to Fourier transformation of $\left\langle\Delta r^{2}(\tau)\right\rangle$, as done in previous studies based on particle tracking and DWS). Equation (6) is often referred to as the generalized Stokes-Einstein equation, and it is valid only under restricted conditions. We shall comment on its applicability to the materials and conditions used in our experiments in our discussion of measurements reported for viscoelastic fluids.

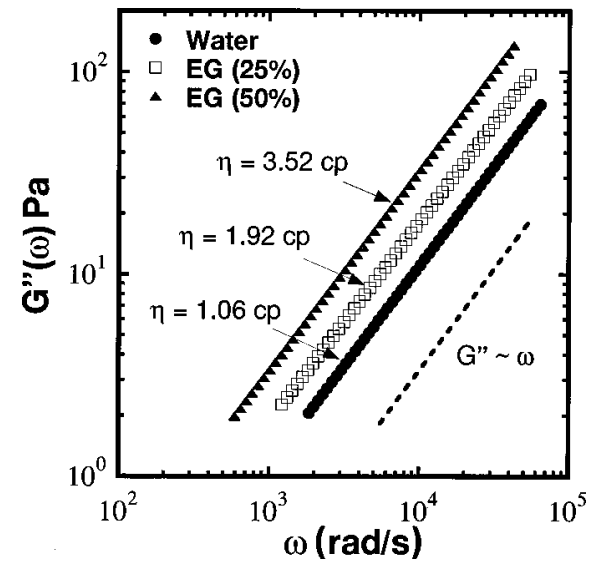

FIG. 1. Loss moduli for water and aqueous solutions of ethylene glycol (EG) at two different concentrations, as indicated. The dashed line indicates the $\omega^{1}$ behavior. The viscosity $\eta$ obtained from the fit is shown for each data set. The corresponding viscosities obtained using a mechanical viscometer are: $\eta=1.02,2.01$, and 3.9 for water and ethylene glycol solutions of concentrations 25 vol \% and 50 vol $\%$, respectively.

\section{EXPERIMENTAL RESULTS AND DISCUSSIONS}

\section{A. Purely viscous fluids}

In order to test the accuracy of our approach, we first report results of a few experiments on simple (i.e., purely viscous) liquids. For this, we use solutions of ethylene glycol (EG) in water at various concentrations and mixed with polystyrene microspheres of diameter $0.2 \mu \mathrm{m}$, at a volume fraction of $10^{-2}$, as probe particles; the experiments were also repeated with beads of $0.1 \mu \mathrm{m}$ in diameter. The bandwidth of the measurement was $2 \mathrm{~Hz}$, and each power spectrum was averaged typically over 100 measurements in order to increase the signal-to-noise ratio. The high-frequency (tail) regions of the power spectra were found to exhibit a power-law dependence of the form $\omega^{n}$, and a least-square analysis of the data with this functional form led to $n=-2 \pm 0.01$ for all the EG solutions and water. Note that this result is what one would expect for the power spectra associated with purely viscous fluids at large frequencies, i.e., for viscous fluids $P(\omega)$ should have a Lorentzian shape, which, for high frequencies, implies a $\omega^{-2}$ dependence. In order to compute the real and imaginary parts of the response function, we used the functional forms obtained from the fit over the frequency domain where they describe the experimental results accurately. This result, in combination with Eqs. (5) and (1), leads directly to $\chi^{\prime \prime}(\omega)$, from which one then obtains the memory component $\chi^{\prime}(\omega)$ using the Kramers-Kronig relation, Eq. (2).

In order to perform the Kramers-Kronig integration in Eq. (2), the power-law dependence of $P(\omega)$ on frequency has been assumed to be valid beyond the actual frequency interval measured. This is a common procedure necessary to avoid the effects of a limited bandwidth. The value of the integral was evaluated for points within the measurement interval. The results obtained are shown in terms of the loss modulus $G^{\prime \prime}(\omega)$ in Fig. 1, for water and EG solutions at two different concentrations. The results shown are for probe par- 


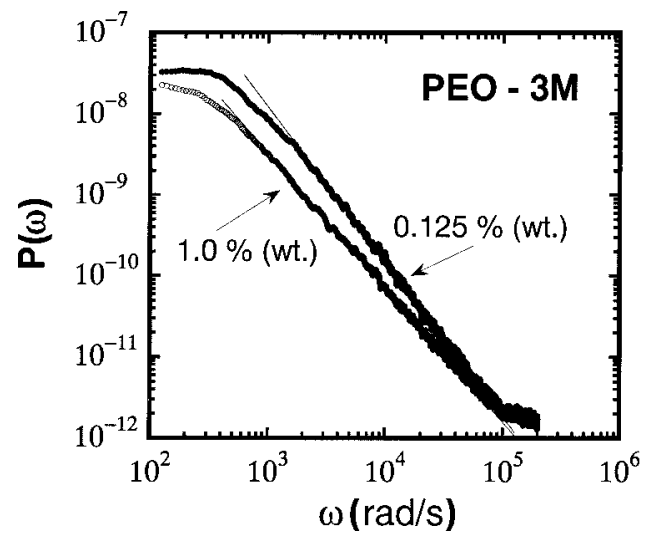

FIG. 2. The raw power spectra for PEO-3M solutions of two different concentrations $(0.125 \%$ and $1 \%$ by weight $)$ obtained using $0.2 \mu \mathrm{m}$ particles.

ticles of diameter $0.2 \mu \mathrm{m}$. A comparison of the results with the line of unit slope shown in the figure demonstrates the linear dependence of $G^{\prime \prime}(\omega)$ in all cases, consistent with what is expected for purely viscous fluids. The intercepts of linear fits of the data give the viscosities of the fluids and are also indicated in Fig. 1. The results obtained $(\eta=1.06,1.92$, and $3.56 \mathrm{cP}$ for pure water and EG concentrations of $25 \%$ and $50 \%$ by volume, respectively) are within $10 \%$ of values measured independently using a mechanical viscometer $(\eta$ $=1.02,2.01$, and $3.9 \mathrm{cP}$, respectively). The corresponding storage moduli $G^{\prime}(\omega)$ are essentially zero, as one would expect for purely viscous materials, and are not shown in the figure.

Not shown here for brevity are the results obtained for the samples with $0.1 \mu \mathrm{m}$ probe particles; however, these results are in excellent agreement with the ones presented in the figure.

It is worth noting that the lower limit of the frequencies accessible in the experiments is determined by the frequency at which the experimental power spectrum deviates significantly from the power function behavior. The upper limit of the frequency interval is set by the measurement noise and does not represent a limitation of the method itself, as the range could be extended with improved instrumentation and noise reduction. In all the experiments, we could obtain the shear modulus over a typical range of at least two orders of magnitude in frequency.

\section{B. Viscoelastic fluids}

We now illustrate the use of the proposed technique for measuring the local response of viscoelastic materials. For this, we use polyethylene oxide (PEO) solutions of various concentrations and molecular weights. PEO is a watersoluble polymer available in a range of molecular weights (e.g., $10^{3}-10^{7}$ ), and its local viscoelastic properties in concentrated solutions have been investigated previously [3]. In the experiments reported in the present paper, all the samples were kept homogenized for several days before the measurements. Figure 2 shows two examples of raw power spectra $P(\omega)$ for aqueous solutions of PEO with an average molecular weight of $3 \times 10^{6}$ (denoted by PEO-3M, where the suffix

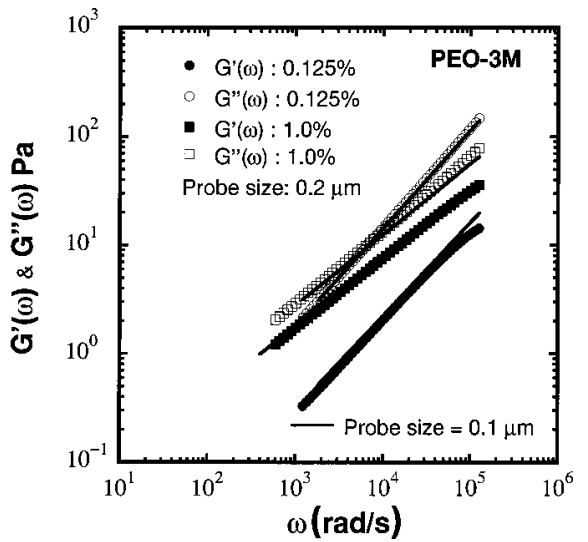

FIG. 3. The storage modulus $G^{\prime}$ and the loss modulus $G^{\prime \prime}$ for PEO-3M solutions at two different concentrations obtained using $0.2 \mu \mathrm{m}$ particles. The results obtained with $0.1 \mu \mathrm{m}$ on the same samples are represented by the solid lines.

$3 \mathrm{M}$ for $3 \times 10^{6}$ identifies the molecular weight) at concentrations of $0.125 \%$ and $1.0 \%$ by weight, as indicated. As evident from Fig. 2, the functional dependence of the measured power spectrum changes from a power of -1.91 $(0.125 \%)$ to $-1.66(1 \%)$ for large frequencies, indicating deviations from the response of a purely viscous fluid [for which one expects a Lorentzian for $P(\omega)$, corresponding to a $\omega^{-2}$ behavior at large frequencies, as discussed in the preceding section].

The above power spectra are converted to $G^{\prime}(\omega)$ and $G^{\prime \prime}(\omega)$ as discussed in the preceding section and the results are plotted in Fig. 3. First, one notes that the relative behavior of $G^{\prime \prime}(\omega)$ and $G^{\prime}(\omega)$ is consistent with what one would expect. In particular, the elastic component is much smaller than the loss modulus for the $0.125 \%$ solution relative to the case of the $1 \%$ solution, since the lower polymer concentration only marginally perturbs the essentially viscous behavior of the solution. Correspondingly, the slope of the loss modulus $G^{\prime \prime}(\omega)$ with respect to the frequency $\omega$ is about 0.91 - a small deviation from a slope of unity expected for purely viscous behavior. For the larger concentration, $G^{\prime}(\omega)$ is noticeably larger than the one for the lower concentration, indicating stronger elasticity. One also notices that the corresponding $G^{\prime \prime}(\omega)$ increases more slowly with $\omega\left(\right.$ as $\left.\sim \omega^{0.66}\right)$ than for the lower concentration. The values for the two concentrations, in fact, intersect each other, but this should be expected. To see this, note that for both solutions $G^{\prime \prime}(\omega)$ should become linear in $\omega$ in the so-called terminal zone, namely, for $\omega \rightarrow 0$, [i.e., $G^{\prime \prime}(\omega) \sim \eta \omega$, where $\eta$ is the effective viscosity of the solution]. The effective viscosity will be higher for the $1 \%$ solution, but the deviation of $G^{\prime \prime}(\omega)$ from linearity with increases in $\omega$ would also be larger for the more concentrated solution. Therefore, the two lines would be expected to intersect at some frequency $\omega \gg 0$ (the precise magnitude being determined by concentration and molecular weight). Finally, the solid lines in Fig. 3 represent the results for the same fluids, now seeded with particles of diameter $0.1 \mu \mathrm{m}$, and the results are almost indistinguishable from the data obtained with $0.2 \mu \mathrm{m}$ particles, suggesting an excellent agreement between the measurements with particles of dif- 
ferent dimensions. The effect of probe size on the interpretation of the data is not a trivial issue, and we shall comment on it below in Sec. III C.

In general, the ratio $G^{\prime \prime} / G^{\prime}=\tan \delta$, known as the loss tangent, provides a measure of the material fluidity of viscoelastic fluids. The real and imaginary parts of $G(\omega)$ for each solution appear to have almost identical dependence on frequency, as suggested by the results shown in Fig. 3; therefore, the loss tangent has a weak dependence on frequency for both concentrations in the range of frequency shown. However, the magnitude of the loss tangent does decrease considerably with polymer concentration, as expected. In general, the loss tangent should approach infinity for a purely viscous material such as water and decrease to zero as the system tends to a perfectly elastic material, such as a gel [17]. We shall return to this in Sec. III D.

In summary, our results show that for viscous fluids the linear dependence of the loss modulus with frequency is recovered from the measured response function and that the values of $G^{\prime \prime}(\omega)$ are very high relative to $G^{\prime}(\omega)$, which is practically zero. When very small quantities of the polymer are added to pure water and the polymer is dispersed homogeneously, the solution remains essentially viscous but the viscosity increases slightly (relative to that of the solvent). If the concentration of the polymer is well below the critical concentration for entanglement, the elastic modulus of the fluid should stay at a very low level, corresponding to a large loss tangent. With subsequent increases in the concentration of the polymer, the fluid will gradually depart from purely viscous behavior until entanglement becomes strong enough. Correspondingly, the fluid would exhibit viscoelastic properties until the concentration of polymer becomes so high that the system becomes essentially a purely elastic solid, characterized by a very small loss tangent and a modulus independent of frequency.

\section{Microrheology versus macrorheology}

To what extent the reported microrheological measurements truly represent the bulk rheology of the materials deserves some attention. It has been recognized in previous studies that the mesh size of a polymer network relative to the size of the probe particle plays a critical role in the overall response of the system to the excitation [2]. It is expected that, whenever the particle size is much smaller than the mesh size, only the response of the fluid is probed, since the cages formed by the polymer network are so large that they do not influence the motion of the particle. Once the particle is comparable in size to the mesh size, both viscous and elastic properties determine the mean-square displacement of the embedded particles. However, the mesh size (relative to the probe size) is not the only relevant parameter, and the question of under what conditions the microrheological measurements of the type reported here accurately represent the macroscopic rheology of the materials requires an examination of other factors. Clearly, this question is relevant in the case of other methods (e.g., particle tracking and DWS) as well, and has been addressed in detail by Levine and Lubensky $[11,12]$. The Levine-Lubensky analysis is based on the so-called "two-fluid" model, in which the mechanical response of a polymer network viscously coupled to an incompressible, Newtonian solvent is examined in the presence of an applied force. In view of the theoretical analysis presented by Levine and Lubensky, our objective here is to simply highlight the important considerations and to establish that our experimental conditions meet the requirements.

The correspondence between microrheology and macrorheology rests on two issues: (i) One is the use of the generalized Stokes-Einstein equation (6) to obtain $G(\omega)$ from the observed response function $\chi(\omega)$. Therefore, under what conditions the use of the generalized Stokes-Einstein relation is acceptable must be addressed. (ii) The second is the influence of any local heterogeneity that might be introduced by the probe particles themselves. We consider each of these separately.

\section{Acceptability of generalized Stokes-Einstein relation}

The acceptability of Eq. (6) rests on three requirements, namely, that the inertial effects of the probe particles, the inertial effects of the fluid, and the longitudinal compression mode of the mesh be all negligible over the frequency range of measurements. The last of the above three requirements arises from the fact that, in contrast to the pure shear applied in macrorheological measurements, the Brownian fluctuations of the probe particle in a microrheological measurement impart a compressional wave on the polymer network (which leads to the draining of the fluid from the denser parts to the more rarified parts). Detailed criteria for all the three requirements have been developed by Levine and Lubensky $[11,12]$ based on certain approximations. Of the three effects, the contribution of particle inertia to $\chi^{-1}(\omega)$ is the easiest to deal with, as it can be obtained from a simple Langevin formulation of the equation of motion for the probe particles (see, for example, Ref. [18]). This contribution is equal to $\omega^{2} m_{p}$ (where $m_{p}$ is the mass of the particle), as also shown by Levine and Lubensky through their more comprehensive analysis. The resulting criterion for the neglect of particle inertia simply requires that the measurement frequencies be restricted to values of $\omega$ such that $6 \pi a G(\omega) \gg \omega^{2} m_{p}$; or, equivalently,

$$
\omega \ll\left(\frac{9 G(\omega)}{2 a^{2} \rho_{p}}\right)^{1 / 2}=\omega_{i, p},
$$

where $\rho_{p}$ is the density of the particle $\left(=1.05 \mathrm{~g} / \mathrm{cm}^{3}\right.$, in the present case). For the present conditions, $\omega_{i, p}$ is of the order of about $10 \mathrm{MHz}$, and therefore the particle inertia may be safely ignored.

In the absence of particle inertia, the two-fluid model leads to an additive expression for compliance that contains, in addition to the Stokes-Einstein term, two additional terms, one corresponding to the fluid inertia and the other for the excitation of the longitudinal degrees of freedom in the system [12]. The criteria for the neglect of fluid inertia include a restriction on $\omega$ similar to the restriction described above,

$$
\omega \ll\left(\frac{\pi^{2} G(\omega)}{4 a^{2} \rho_{f}}\right)^{1 / 2}=\omega_{i, j},
$$


where $\rho_{f}$ is the fluid density $\left(=1 \mathrm{~g} / \mathrm{cm}^{3}\right)$, and a restriction on the mesh size $\xi$ of the polymer network

$$
\varepsilon=\left(\frac{\xi}{a}\right)^{2} \ll 1
$$

Equation (8) arises from a comparison of the inertial decay length with the particle radius. In view of the fact that $\rho_{f}$ is comparable to $\rho_{p}$ and that the equations for $\omega_{i, f}$ and $\omega_{i, p}$ are similar, $\omega_{i, f}$ is also of the order of $10 \mathrm{MHz}$. We shall see shortly that the condition on $\xi$ is also easily met in our experiments, so that the effects of fluid inertia are negligible in the range of frequencies over which we report $G^{\prime}(\omega)$ and $G^{\prime \prime}(\omega)$.

The criterion for the neglect of longitudinal compressional mode is also discussed by Levine and Lubensky in detail. In particular, they show that for frequencies $\omega>\omega_{c}$ ( $\omega_{B}$ in the notation of Levine and Lubensky), the polymer network locks in viscously with the incompressible solvent, thus eliminating the free-draining, longitudinal modes. Therefore, for frequencies larger than $\omega_{c}$, which is of the order of $10 \mathrm{~Hz}$ [12], the microrheological measurements fall into closer correspondence with macrorheological response of the system.

Before addressing the remaining consideration of the role of local heterogeneities introduced by the probe particles themselves, it is necessary to develop estimates for the mesh size $\xi$ of the polymer network in terms of the polymer concentration. First, we note that the radius of gyration $R_{g}$ for the PEO molecules in dilute solutions may be estimated from the experimental work of Devanand and Selser [19]. The values are about $130 \mathrm{~nm}$ for PEO-3M and $230 \mathrm{~nm}$ for PEO-8M (i.e., PEO of molecular weight $8 \times 10^{6}$ ). The free coils in a dilute solution begin to overlap at an overlap concentration $c^{*}$ given by

$$
c^{*} \approx \frac{3 M}{4 \pi N_{A} R_{g}^{3}},
$$

where $M$ is the molecular weight and $N_{A}$ is Avogadro's number. The above equation follows from the definition of the overlap concentration, namely, the concentration at which the segment density in the solution equals the segment density within each coil. In the present paper, the overlap concentrations are roughly $0.05 \mathrm{wt} \%$ for PEO-3M and 0.03 wt $\%$ for PEO-8M. At and beyond the overlap concentration, the solution can be described as an un-cross-linked network of mesh size $\xi$, which scales with concentration as (see de Gennes [20])

$$
\xi \approx R_{g}\left(\frac{c}{c^{*}}\right)^{-3 / 4}
$$

where $c$ is the polymer concentration. One sees immediately that except for concentrations very close to $c^{*}$ the ratio $\varepsilon$,

$$
\varepsilon=\left(\frac{\xi}{a}\right)^{2} \approx\left(\frac{R_{g}}{a}\right)^{2}\left(\frac{c}{c^{*}}\right)^{-3 / 2},
$$

meets the condition $\varepsilon \ll 1$ required for the neglect of fluid inertia. For instance, for the 1\% PEO-3M for which results are shown in Fig. 3, $\varepsilon$ is roughly 0.02 for the $0.2-\mu \mathrm{m}-$ diameter probe particle $(a=0.1 \mu \mathrm{m})$ and is about 0.08 for the $0.1-\mu \mathrm{m}$-diameter probe. For the lower concentration shown $(0.125 \%)$, the condition is not met for the $0.1-\mu \mathrm{m}$ diameter probe particle (although the other criteria on the frequencies are easily satisfied). However, at this low concentration, the solution is expected to deviate only marginally from the pure-solvent case (as discussed earlier in this section), and a comparison of the data for pure water in Fig. 1 with the results in Fig. 3 for $0.125 \%$ with the $0.1-\mu \mathrm{m}$ diameter probe in fact reveals that the values of $G^{\prime \prime}(\omega)$ are in the expected range.

\section{Local heterogeneity introduced by the probe particles}

We are now in a position to comment on the effects of any local heterogeneities introduced by the probe particles themselves. If one takes the spatial range of inhomogeneity (from the surface of the probe particle) to be $\left(a^{\prime}-a\right)$, the measurements of $\chi(\omega)$ [equivalently $G(\omega)$ ] then will represent spatially averaged values in a system with material parameters for distances beyond $a^{\prime}$ corresponding to bulk values and the ones below $a^{\prime}$ differing from bulk values because of local inhomogeneities. Levine and Lubensky [11] have addressed this problem and showed that, as one would expect, microrheological measurements would reflect the bulk rheological behavior for

$$
\left(\frac{a^{\prime}-a}{a}\right) \ll 1,
$$

i.e., if the range of spatial inhomogeneity is small relative to the probe radius $a$. One would expect the spatial range ( $a^{\prime}$ $-a$ ) of the disruption of the homogeneity of the network to be roughly $\left(a^{\prime}-a\right) \sim \alpha \xi$, with the magnitude of the proportionality constant $\alpha$ being of the order of 1 . Therefore, in view of the discussion concerning the relative mesh size $\varepsilon^{1 / 2}=(\xi / a)$ above, it follows that the condition specified by Eq. (13) is also easily met in all but one case in our experiments (i.e., except in the case of polymer concentrations close to $c^{*}$ for the lower probe size). Where inhomogeneity is an issue, two-particle (i.e., cross-correlation) microrheology is a better alternative in place of single-particle microrheological measurements [10]. The fact that we obtain physically consistent values for $G(\omega)$ even in the one limiting case is interesting and might be the result of the relative flexibility of the PEO chains (as opposed to the stiffer $f$-actin networks, for which one-particle microrheology leads to noticeably different results from the bulk rheology probed using two-particle method [10]) and the weak dependence of the mechanical behavior of the network to relatively small deviations from homogeneity in the bulk.

\section{Liquid-gel transitions}

In order to examine the method across liquid-gel transition, we performed systematic experiments on PEO solutions over a broad range of concentrations. The experiments were 

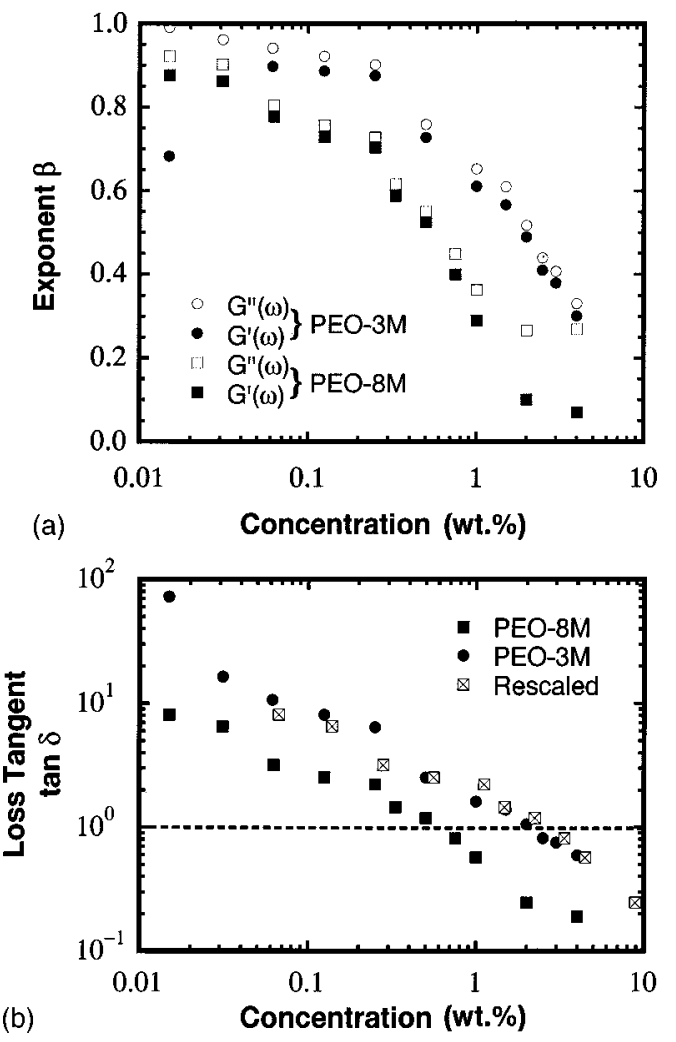

FIG. 4. The exponent $\beta$ and loss tangent $\tan \delta$ as functions of the PEO concentrations for two molecular weights, namely, 3 $\times 10^{6}$ (PEO-3M) and $8 \times 10^{6}$ (PEO-8M). (a) The power-law dependence of $G^{\prime}$ and $G^{\prime \prime}$ on frequency, as indicated; the parameter $\beta$ was obtained by fitting $G^{\prime}(\omega)$ and $G^{\prime \prime}(\omega)$ with the function $\alpha \omega^{\beta}$. (b) The loss tangent for the same solutions as in Fig. 4(a) measured as the ratio $G^{\prime \prime}(\omega) / G^{\prime}(\omega)$ corresponding to an angular frequency of $10^{4} \mathrm{rad} / \mathrm{s}$. The "rescaled" data points correspond to the data for PEO-8M when the corresponding concentrations are multiplied by 4.25 .

performed on aqueous solutions of PEO with two molecular weights: $3 \times 10^{6}$ (PEO-3M) and $8 \times 10^{6}$ (PEO-8M), with concentrations by weight from $0.0125 \%$ to $4 \%$. The results for the shear modulus and loss tangent are summarized in Figs. 4(a) and 4(b). As in the case of the results shown in Fig. 3, both $G^{\prime}(\omega)$ and $G^{\prime \prime}(\omega)$ vary as $\alpha \omega^{\beta}$, with different prefactors $\alpha$ and exponents $\beta$. Figure 4(a) presents the exponents $\beta$ for $G^{\prime}(\omega)$ and $G^{\prime \prime}(\omega)$ and shows that beyond the overlap concentrations the exponents for $G^{\prime}(\omega)$ and $G^{\prime \prime}(\omega)$ are essentially the same [thereby indicating that the loss tangent, shown in Fig. 4(b), is a very weak function of $\omega$ for the solutions considered]. It can be seen from Fig. 4(a) that for the lower molecular weight PEO (PEO-3M), viscous behavior dominates in the region of low polymer concentrations. This region is characterized by an almost linear frequency dependence of $G^{\prime \prime}(\omega)$, while $G^{\prime}(\omega)$ is small in magnitude (large $\tan \delta$ ). At the other end of the concentration interval considered, the storage modulus becomes larger than the loss modulus, indicating gelation. As an example, Fig. 5 presents $G^{\prime}(\omega)$ and $G^{\prime \prime}(\omega)$ for the heavier molecule (PEO-8M); $G^{\prime}(\omega)$ is almost constant with respect to the frequency, while the associated loss tangent decreases to values signifi-

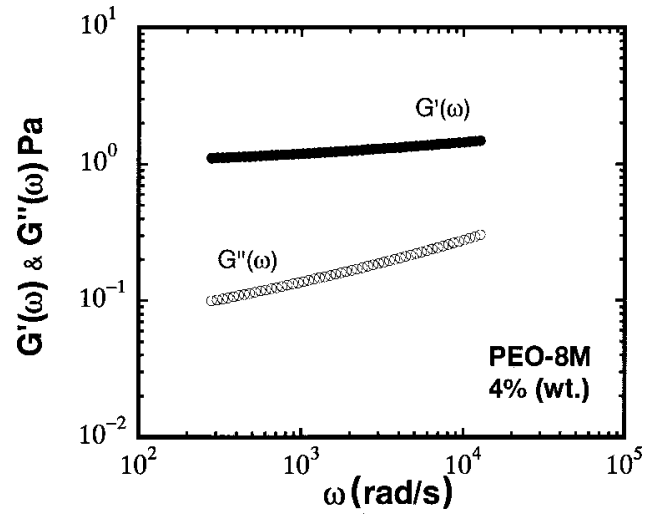

FIG. 5. The storage modulus $G^{\prime}$ and the loss modulus $G^{\prime \prime}$ for PEO-8M solution at a concentration of $4 \mathrm{wt} \%$, i.e., beyond the gelation point.

cantly smaller than unity. This transition to a gel-like behavior is less developed for the PEO-3M solutions, which require higher concentrations for the liquid-to-gel transition [21].

\section{E. Microrheology at interfaces of complex systems}

A particular advantage of the experimental arrangement and technique we propose here is the possibility of probing changes in viscoelasticity through an interface as a function of spatial position within the interface. That is, so far, we have demonstrated the ability of the technique to measure the rheological properties of complex fluids within small coherence volumes, and it has been shown that detailed information about the liquid-gel transition can be obtained with the approach proposed. The information about the fluid under investigation is retrieved from a measurement volume of the order of a tenth of a picoliter. This unique feature of our technique makes the method appealing for investigating systems with heterogeneities existing on a much smaller scale than could be measured with any other method. To illustrate this, we consider a test system with a gradient in the constitutive properties along one spatial coordinate, created by placing two different solutions in contact so that an interfacial region of about $2000 \mu \mathrm{m}$ is formed. More specifically, an aqueous solution of ethylene glycol ( $25 \%$ by volume) was placed in contact with a polymer solution of PEO-8M $(0.5 \%$ by mass) in a capillary tube of $1 \mathrm{~mm}$ diameter. The optical fiber (125 $\mu \mathrm{m}$ diameter) was then immersed in the liquid and successive measurements were taken at various depths, along the spatial concentration gradient created. The results for the loss tangent as a function of depth $z$ are summarized in Fig. 6. The figure demonstrates that there is a clear transition from a region of high loss-tangent values associated with the viscous liquid (the ethylene glycol solution) to the low values characteristic of viscoelastic fluids (PEO-8M); the transition occurs over a distance of about $2000 \mu \mathrm{m}$. It can be seen that at the two extreme ends (above and below the interface), the values of the loss tangent show almost no dependence on $z$, thereby identifying homogeneity at the two extremes. The corresponding values of the loss tangent are in excellent agreement with the ones measured for each fluid 


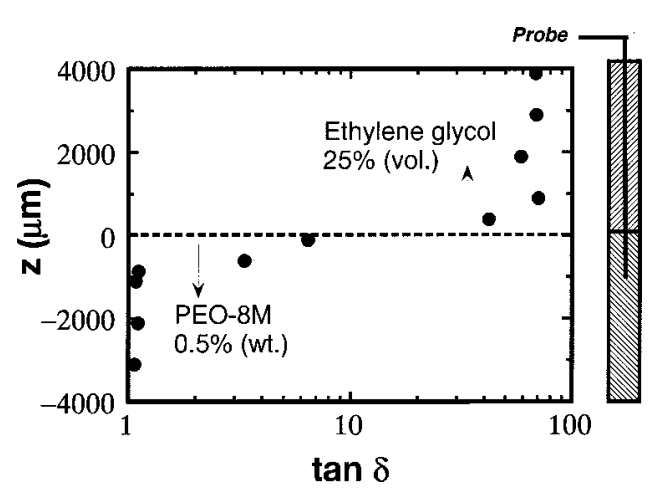

FIG. 6. Loss tangent (measured as in Fig. 4) as a function of $z$ coordinate, as the optical fiber goes from a layer of simple viscous liquid ( $25 \mathrm{vol} \%$ of ethylene glycol) to a viscoelastic fluid $(0.5 \mathrm{wt} \%$ of PEO-8M).

independently. This remarkable result proves that the optical fiber slips through the fluid, without substantially carrying solute molecules from one solution to the other. The above results imply that the proposed technique can be used to obtain a three-dimensional mapping of the microrheological properties in highly heterogeneous complex fluids, thereby opening a new class of potential applications.

\section{CONCLUSIONS}

We have introduced an optical technique capable of measuring the high-frequency behavior of the shear modulus associated with complex fluids. Owing to the coherence properties of the light used, a small measurement volume of the order of picoliters is isolated at the end of a single-mode optical fiber and the data are analyzed directly in the frequency domain. The coherence volume used here is, to our knowledge, the smallest accessible to rheological measurements to date, and should lead to important new applications, especially in investigations of heterogeneous biomaterials. In addition, the minute dimensions of the measurement volume suppress multiple scattering and, therefore, the technique can be applied to fluids having a broad range of optical densities. It should be stressed that the above, extremely small measurement volume is obtained purely by optical means without requiring that one physically isolates a small quantity of the material to be tested. It has been shown that the method has the ability to infer the complex shear moduli for a variety of systems and also to monitor morphological transformations in complex fluids, such as the liquid-gel transition. The use of optical fibers ensures experimental flexibility. Finally, the technique can be used to build three-dimensional maps of rheological properties in heterogeneous systems, and thus opens a whole new field of microrheological applications.
[1] F. C. MacKintosh and C. F. Schmidt, Curr. Opin. Colloid Interface Sci. 4, 300 (1999).

[2] F. Amblard, A. C. Maggs, B. Yurke, A. N. Pargellis, and S. Leibler, Phys. Rev. Lett. 77, 4470 (1996).

[3] T. G. Mason, K. Ganesan, J. H. van Zanten, D. Wirtz, and S. C. Kuo, Phys. Rev. Lett. 79, 3282 (1997).

[4] F. Gittes, B. Schnurr, P. D. Olmsted, F. C. MacKintosh, and C. F. Schmidt, Phys. Rev. Lett. 79, 3286 (1997).

[5] T. G. Mason and D. A. Weitz, Phys. Rev. Lett. 74, 1250 (1995)

[6] H. Ma, J. Jimenez, and R. Rajagopalan, Langmuir 16, 2254 (2000)

[7] F. Gittes and F. C. MacKintosh, Phys. Rev. E 58, R1241 (1998).

[8] D. C. Morse, Phys. Rev. E 58, R1237 (1998).

[9] J. Xu, A. Palmer, and D. Wirtz, Macromolecules 31, 6486 (1998)

[10] J. C. Crocker, M. T. Valentine, E. R. Weeks, T. Gisler, P. D. Kaplan, A. G. Yodh, and D. A. Weitz, Phys. Rev. Lett. 85, 888 (2000).

[11] A. J. Levine and T. C. Lubensky, Phys. Rev. Lett. 85, 1774 (2000).

[12] A. J. Levine and T. C. Lubensky, Phys. Rev. E 63, 041510 (2001)

[13] G. Popescu and A. Dogariu, Opt. Lett. 26, 551 (2001).

[14] The sensitivity of the technique is enhanced due to several reasons. First, the backscattered intensity is a product of the field amplitude of the scattered light and that of the Fresnel reflection. Therefore, the specular reflection amplifies the backscattering signal, as noted in the text. In addition, as in any heterodyne technique, the dynamic range is increased since the measured intensity is proportional to the squared root of the backscattered intensity and not to the intensity itself. This is not the case in traditional DLS and DWS measurements. Second, although the incident beam undergoes only single scattering due to the reduced dimensions of the coherence volume, the total detected signal is the result of backscattering from a collection of particles. Lastly, because the measurements are made directly in the frequency domain, the electrical noise is reduced dramatically by narrowing the bandwidth of the measurement and by extensive averaging.

[15] D. A. Weitz, J. X. Zhu, D. J. Durian, H. Guang, and D. J. Pine, Phys. Scr. 149, 610 (1993).

[16] The actual data analysis proceeds as follows. We first normalize the area under the power spectrum $P(\omega)$ by fitting the low-frequency region with a polynomial and extrapolating the polynomial to $\omega=0$. Doing so guarantees the availability of the full spectrum of frequencies in the normalization. Once $P(\omega)$ is normalized the subsequent data analysis follows the procedure discussed in the text.

[17] R. I. Tanner, Engineering Rheology, 2nd ed. (Oxford University Press, Oxford, UK, 2000).

[18] R. Rajagopalan, Colloids Surf. A 174, 253 (2000).

[19] K. Devanand and J. C. Selser, Macromolecules 24, 5943 (1991).

[20] P.-G. de Gennes, Scaling Concepts in Polymer Physics (Cornell University Press, Ithaca, NY, 1979).

[21] The results shown in Fig. 4(b) appear to show an interesting scaling behavior. As shown in the figure, the loss tangents of the lower-molecular-weight solution correspond to those of the higher-molecular-weight solution for concentrations that are roughly 4.25 times the concentrations of the latter. 